\title{
Optimized Design of a Rigid Kinematic Module for Antagonistic Soft Actuation
}

\author{
Ming Luo, Erik H. Skorina, Weijia Tao, Fuchen Chen, and Cagdas D. Onal
}

\begin{abstract}
Soft actuators can be useful in human-occupied environments because of their adaptable compliance and light weight. We previously proposed a new actuator: the reverse pneumatic artificial muscle (rPAM), and developed an analytical model to predict its performance both individually and while driving a 1 degree of freedom revolute joint antagonistically. Here, we expand upon this previous work, adding a correction term to improve model performance and using it to perform optimization on the kinematic module dimensions to maximize achievable joint angles. We also offer advances on the joint design to improve its ability to operate at these larger angles. The new joint had a workspace of around $\pm 60^{\circ}$, which was predicted accurately by the improved model.
\end{abstract}

Index Terms-Soft Actuation, Pneumatics, Analytical Modeling

\section{INTRODUCTION}

Soft actuators offer many advantages over traditional rigid motors. Robots operated by traditional motors can be dangerous to humans because of their rigidly and weight, which often constrains their ability to operate efficiently in human-trafficked environments. Soft actuators, on the other hand, can absorb energy to enable safe and adaptive physical interaction with the environment. In addition, they operate in a similar way to biological muscles, allowing for a bioinspired approach to robotics and actuation.

Our previous work [1], [2] presents an innovative approach to soft actuation consisting of an actuator we call the Reverse Pneumatic Artificial Muscle (rPAM) which is based on the design of the McKibben muscle [3], the fiber reinforced elastomeric enclosure (FREE) [4] and the fluidic elastomer actuator (FEA) [5]-[9]. Compared with traditional rigid actuators [10]-[12], the rRAM is lighter, allowing it be carried in robotic limbs easier, and more compliant, allowing it to safely operate around humans. This actuator consists of a single cylindrical pressure chamber molded our of silicone and reinforced radially by fibers wound in two symmetrical small-angle helices to approximate a series of circles along the length. The threads counteract the hoop stresses in the pressure chamber. This is similar to the McKibben actuator, only the rPAM extends rather then contracts when pressurized. The resulting actuators are easier to fabricate and can provide large forces due to a larger range of input pressures.

Previously we presented novel numerical and analytical models for predicting actuator behavior, and compare model

The authors are with the Mechanical Engineering Department and Robotics Engineering Program, Worcester Polytechnic Institute, MA 01609, USA. All correspondence should be addressed to Cagdas D. Onal cdonal@wpi.edu
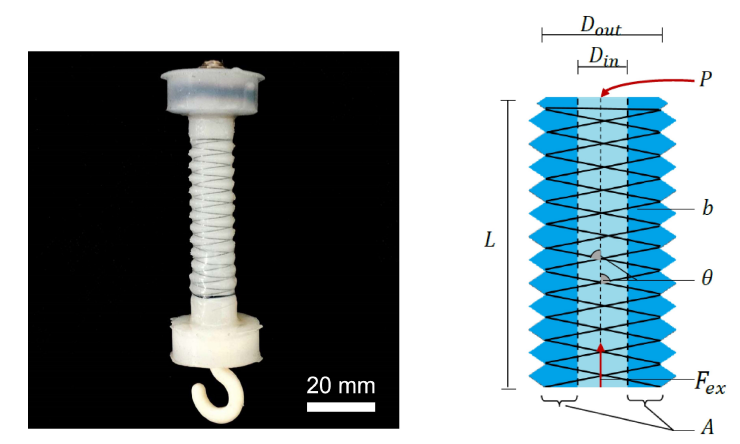

Fig. 1. A physical prototype (Left) of the proposed soft linear actuator, called reverse pneumatic artificial muscle (rPAM), which offers convenient physical and fluidic connectors to operate rigid kinematic linkages. Geometric parameters of the rPAM after deformation (right)

predictions with experimental actuator deformations under a range of payloads (up to $1.3 \mathrm{~kg}$ ) to determine accuracy and limitations of both models. Both models incorporate hyperelastic properties of the silicone rubber used in rPAM fabrication via the Ogden hyperelastic solid model. In addition, we studied the application of rPAMs to driving kinematic linkages. We used the analytical model to predict the response 1-Degree of Freedom revolute joint driven by two rPAMs in antagonism, and verified it through experimentation.

However, we note two limitations of the previous work. First, the analytical model could not predict actuator response under a heavy load, because the helical constraint force is only a function of pressure which could not capture the initial force under large payload. Second, the workspace of 1-DoF revolute joint ( -18 to 18 degree) was narrow at the fixed pressure range (41 to $96 \mathrm{kPa}$ ). This was caused in part by the sub-optimal nature of the joint dimensions. Larger rotation angles would cause the ends of the actuators to become misaligned, forcing them to bend slightly. This bending can become pronounced at higher pressures, potentially leading to reductions in actuator performance.

In this paper we introduce a correction factor based on experimental data in order to reduce the model error under large weights and prestrain values. We also use the model to optimize the dimensions of the 1-DoF revolute joint in order to maximize its workspace.

The contributions of this work include:

- The addition of a correction factor to allow our analytical model to function at under larger payloads.

- The optimization of the 1-DoF revolute joint to maximize the operating range. 


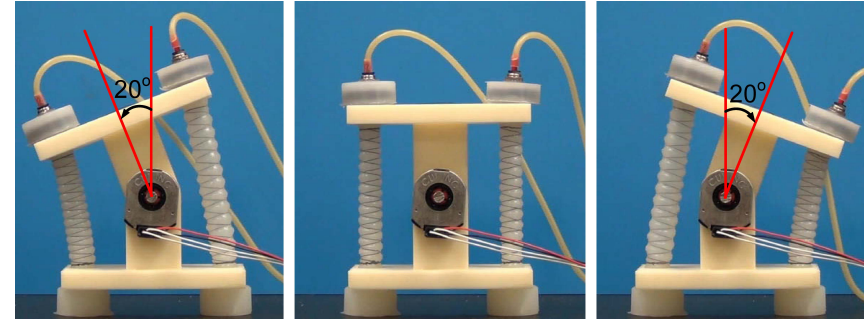

Fig. 2. The original rPAM actuated 1-DoF revolute joint. It is shown here at rest and when actuated in both directions.

\section{Previous Work}

\section{A. Soft Actuator Fabrication and Modeling}

The presented soft linear actuator (rPAMs) was made by molding silicone rubber (Smooth-on Dragonskin 10) in a 3$\mathrm{D}$ printed mold and twisted by the double helix nylon wire (Fig. 1). The right panel of Figure 1 shows the parameters used in the analytical model. Where rPAMS pressurized, it generates two different kind forces: The helical constraint force $F_{\text {cons }}$ and the internal material stress force $F_{\text {int }}$ :

The force balance in steady state as:

$$
F_{\text {ext }}=F_{\text {cons }}+F_{\text {int }},
$$

Where $F_{\text {ext }}$ is the external force.

The helical constraint force:

$$
F_{\text {cons }}=P\left(\frac{3 \frac{L^{2}}{b^{2}}-1}{4 \pi n^{2}}-\frac{A_{o}}{\lambda}\right),
$$

Where the principal stretch $\lambda=\frac{L}{L_{o}}=\frac{A_{o}}{A}$ for incompressible material [13].

The internal material stress force

$$
F_{\text {int }}=\sum_{i=1}^{3} \frac{2 \mu_{i} A_{o}}{\alpha_{i}}\left(\lambda^{\alpha_{i}-1}-\lambda^{-\frac{\alpha_{i}}{2}-1}\right)
$$

where where $\mu_{i}$ and $\alpha_{i}$ are material constants obtained by experimental tensile testing data in Ogden model [14]

\section{B. Joint Design}

We previously developed a simple 1-DoF revolute joint. As the rPAMs only work in extension, bidirectional operation necessitates the use of two actuators in antagonism. The final prototype can be seen in Figure 1. Figure 3 displays both the analytical and experimental rotation angle of the joint to combinations of pressure inputs for both rPAMs between 41 and $96 \mathrm{kPa}$.

In testing, this original joint design still suffered problems. At larger angles, the actuators collided with parts of the joint, reducing mobility. In addition, the actuators had fixed mounts on the joint. These mounts would rotate out of alignment with each other as the joint rotated, causing bending and inconsistencies with the analytical model.

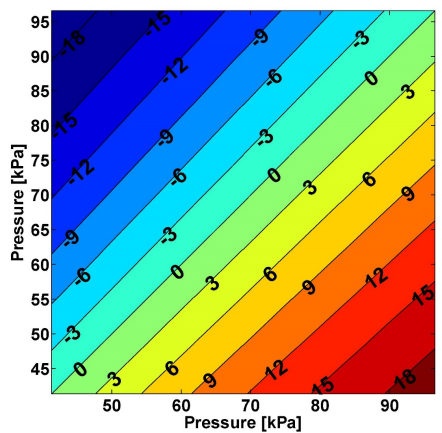

(a) Simulation

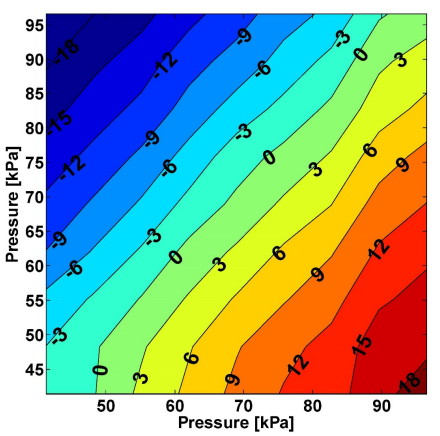

(b) Experiment
Fig. 3. Comparison of model predictions and experimental results for a range of input pressures ranging from 41 to $96 \mathrm{kPa}$ for both rPAMs. Contour plots of the resulting steady-state joint angle in degrees are displayed for simulation (a) and experimenta (b) results. Joint angle values are stepped at 3 degrees, annotated on the curves, and indicated as color coding from blue to red.

\section{Design AND ANALysis of the IMPROVED Kinematic Skeletal Module}

In order to mitigate the bending that was occurring in the original rPAM-driven kinematic module, we decided to modify the way the actuators were mounted on the joint. Instead of mounting them directly on the upper and lower plates of the joint, we mounted them on separate plates that were connected to the rest of the module by a pin joint. This would allow the actuators to swivel as the main joint rotated, maintaining their alignment without needing to bend.

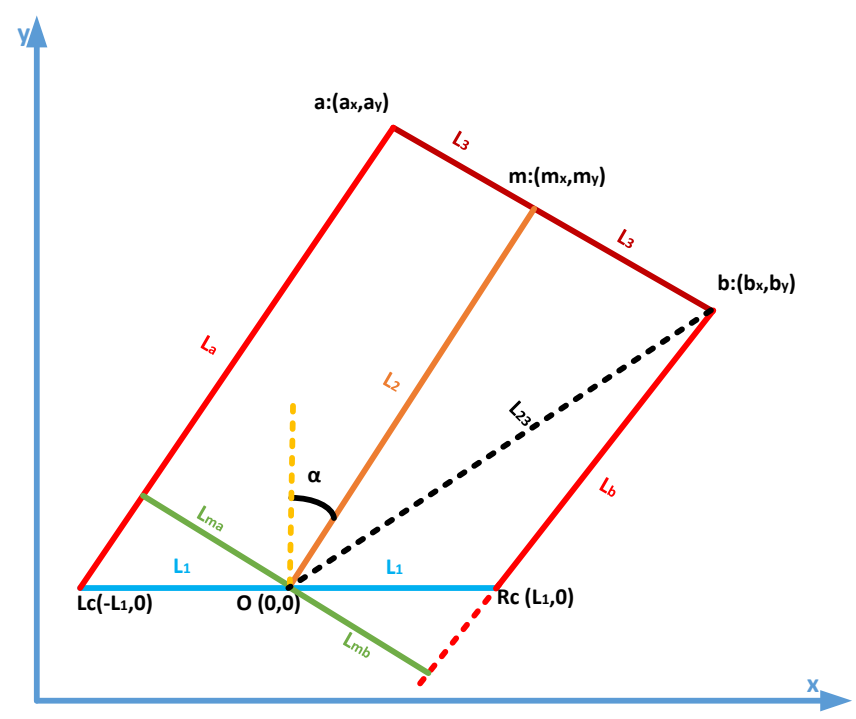

Fig. 4. The geometric model of the proposed 1-DoF joint setup. The blue,brown and dark lines represent the rigid joint links, while the red lines represent the rPAMs. The green lines are the calculated moment arms for each soft actuator.

Preliminary optimization work indicated that the joint should be located along the bottom plate to maximize the workspace and minimize self-collision. Modeling and optimization is performed with this assumption.

Simulations utilize the coordinate information given in 

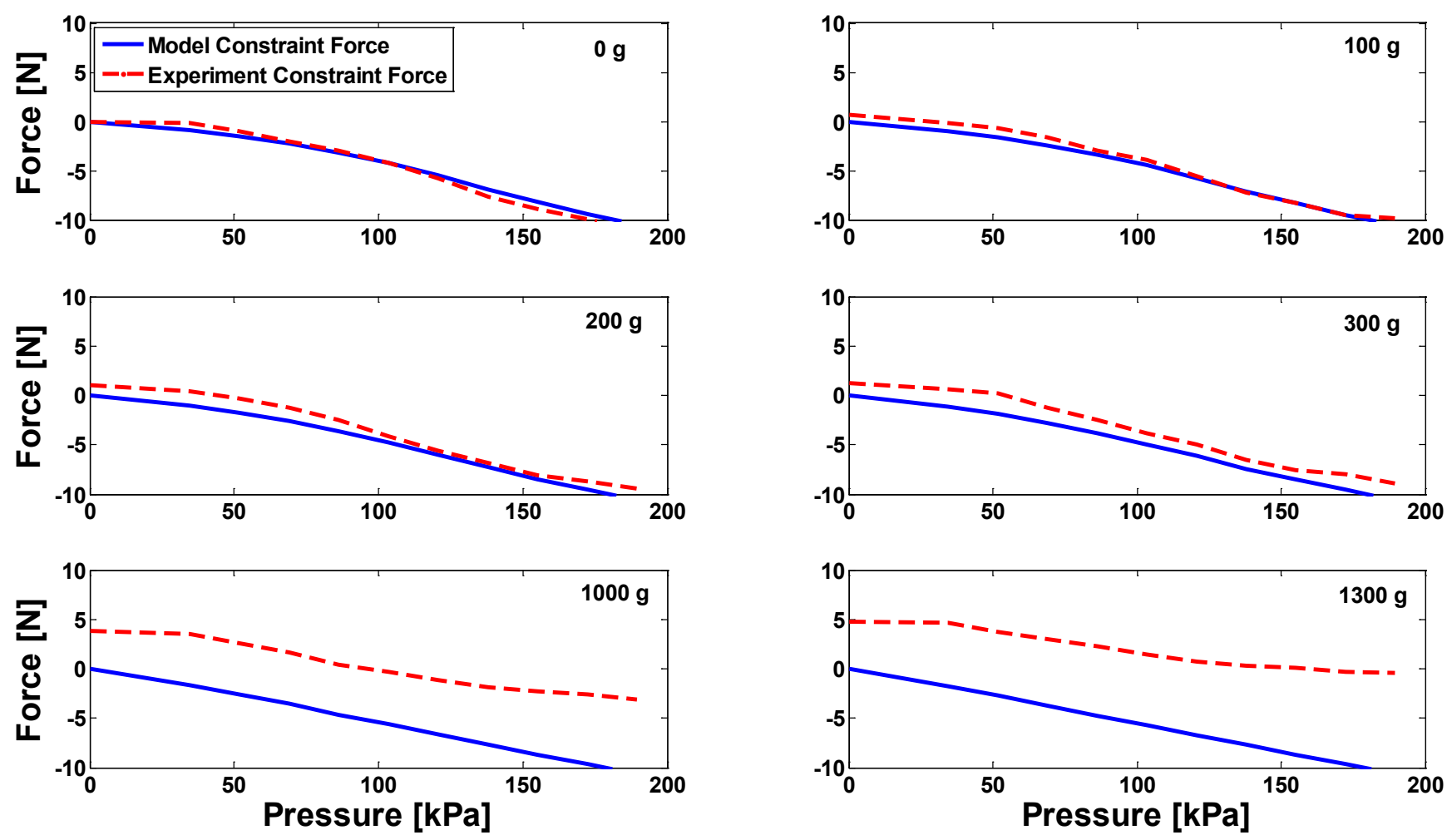

Fig. 5. Experimental (red dashed curve), analytical (blue solid curve) helical constraint forces for six different external payloads of $0,100,200,1000$ and $1300 \mathrm{~g}$ under input pressures of 0 to $190 \mathrm{kPa}$.

Figure 4 for each significant point on the 1-DoF revolute joint kinematic model. Using these relations, the lengths of the two actuators are given as:

$$
\begin{aligned}
L_{a}= & \sqrt{\left(a_{x}+L_{1}\right)^{2}+a_{y}{ }^{2}} \\
& =\sqrt{{L_{1}}^{2}+{L_{2}}^{2}+{L_{3}}^{2}+2 L_{1} L_{2} \sin \alpha-2 L_{1} L_{3} \cos \alpha} \\
L_{b}= & \sqrt{\left(b_{x}-L_{1}\right)^{2}+b_{y}{ }^{2}} \\
= & \sqrt{{L_{1}}^{2}+{L_{2}}^{2}+{L_{3}}^{2}-2 L_{1} L_{2} \sin \alpha-2 L_{1} L_{3} \cos \alpha}
\end{aligned}
$$

$L_{m a}, L_{m b}$ are the moment of the actuator $\mathrm{A}$ and $\mathrm{B}$. The $L_{m a}, L_{m b}$ is the height of the triangle $O a L c$ and $O b R c$ respect to the side $a L c$ and $b R c$. According to the Heron's formula [15]:

$$
\begin{aligned}
L_{m a} & =\frac{2 \sqrt{S_{a}\left(S_{a}-L_{1}\right)\left(S_{a}-L_{23}\right)\left(S_{a}-L_{a}\right)}}{L_{a}} \\
L_{m b} & =\frac{2 \sqrt{S_{b}\left(S_{b}-L_{1}\right)\left(S_{b}-L_{23}\right)\left(S_{b}-L_{a}\right)}}{L_{b}},
\end{aligned}
$$

where $S_{a}=\frac{L_{1}+L_{23}+L_{a}}{2}, S_{b}=\frac{L_{1}+L_{23}+L_{b}}{2}$

When the joint reaches steady-state balance, the two external moments exerted by actuators A and B should be
TABLE I

EQUATIONS FOR THE COORDINATES OF SIGNIFICANT POINTS IN THE 1-DoF REVOLUTE JOINT MODEL.

\begin{tabular}{lll}
\hline \hline Point & X coordinate & Y coordinate \\
\hline$m$ & $L_{2} \sin \alpha$ & $L_{2} \cos \alpha$ \\
$a$ & $L_{2} \sin \alpha-L_{3} \cos \alpha$ & $L_{2} \cos \alpha+L_{3} \sin \alpha$ \\
$b$ & $L_{2} \sin \alpha+L_{3} \cos \alpha$ & $L_{2} \cos \alpha-L_{3} \sin \alpha$ \\
\hline
\end{tabular}

equal:

$$
\begin{aligned}
& F_{\text {exta }} L_{m a}=F_{\text {extb }} L_{m b}, \\
& \left(F_{\text {inta }}+F_{\text {consa }}\right) L_{m a}=\left(F_{\text {intb }}+F_{\text {cons } b}\right) L_{m b},
\end{aligned}
$$

where $F_{\text {exta }}, F_{\text {extb }}$ are the external force of the actuator A and B. $F_{\text {inta }}, F_{\text {intb }}$ are the internal force of the actuator A and B. $F_{\text {consa }}, F_{c o n s b}$ are the constraint force of the actuator $\mathrm{A}$ and $\mathrm{B}$.

\section{RESUlts AND Discussion}

In the original joint prototype, the actuators were prestrained for $30 \%$ of the original length, which is equivalent to a small stretch force when compared to the other two forces. However, if the stretched length increased up more than $30 \%$. The initial stretch force might not be negligible. It is challenging to model the initial stretch force physically, because the material property of the nylon thread combined with the helical geometry is not straightforward. Therefore, we adopt an empirical approach to determine the relation between the initial stretch and the corresponding forces. 


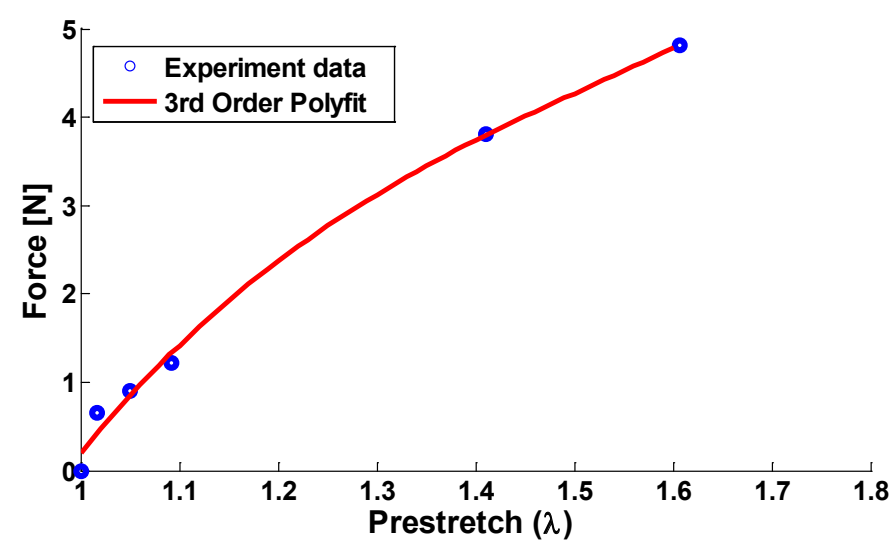

Fig. 6. The experimental stretch force with respect to the prestretch (blue circles) and a third order polynomial fit (red solid curve).

Figure 5 shows the single actuator helix constraint force from the analytical model and the experiment based on the experimental length under $0 \mathrm{~g}, 100 \mathrm{~g}, 200 \mathrm{~g}, 300 \mathrm{~g}, 1 \mathrm{~kg}$ and $1.3 \mathrm{~kg}$ payload. The analytical model constraint force can be calculated directly from the helix constraint force using (2). The experimental constraint force is calculated by the internal force (3) and the force balance (1). When the payload is heavier, the difference in initial stretch force between the two kinds of the helical constraint is more obvious. In order to reduce the analytical model error caused by the initial stretch force, we incorporated an empirical correction term. Figure 6 displays the experimental data of the changing length ratio $\lambda$ and the stretch force as well as its 3rd order polynomial fit curve. Therefore, the force balance equation can be rewritten as:

$$
F_{\text {ext }}=F_{\text {cons }}+F_{\text {int }}+F_{\text {stretch }},
$$

where $F_{\text {stretch }}$ is the initial stretch force.

In order to improve the performances of the actuator, the proposed joint mechanism needs to be optimized. The main objective of the optimization is to maximize the rotation angle of the joint when the input pressure is fixed. For the optimization algorithm, the length of each rigid link are the design variables. The rotation angle $\alpha$ could be determined by the rigid link lengths and the length of both antagonistic actuators. The constraint $g_{1}$ is the torque balance of the joint from (6). The constraint $g_{2}$ and $g_{4}$ shows the boundary of the link $L_{1}, L_{3}$. The lower boundary of the $g_{2}$ and $g_{4}$ determined by the size of the central joint and the actuator swivel joints. The constraint $g_{3}$ is the limitation of the link $L_{2}$. The range of the length of the soft actuator is from $0.07 \mathrm{~m}$ to $0.15 \mathrm{~m}$. In order to keep the soft actuators from deforming too much, the upper boundary is was set to $0.14 \mathrm{~m}$. We used a genetic algorithm to find the optimal solution in Matlab. The optimal result happens when $L_{1}$ is chosen at the lowest boundary and $L_{3}$ chosen at the highest boundary.

The optimization parameters fed to the genetic algorithm were as follows:

Design variables:

$L_{1}, L_{2}$ and $L_{3}$

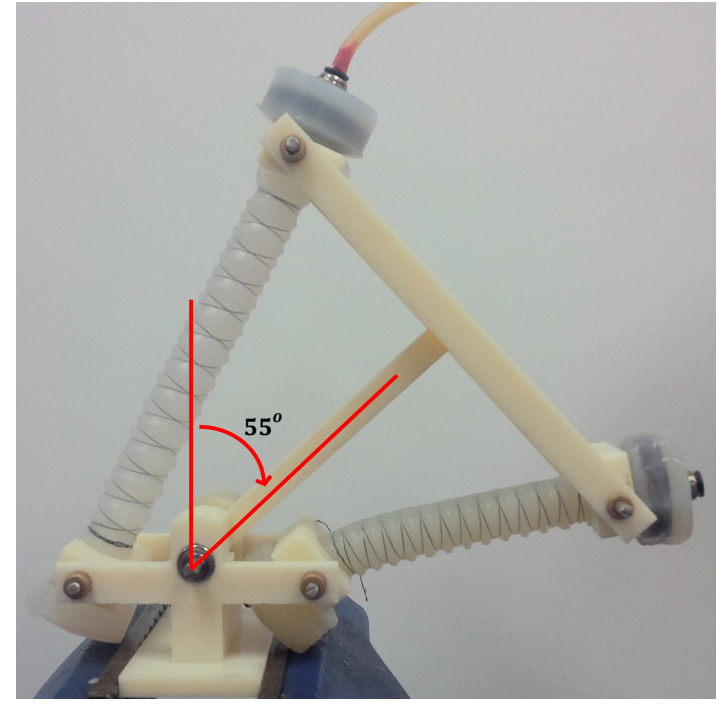

Fig. 7. The final joint design. Left actuator is pressurized to $138 \mathrm{kPa}$ and the right actuator is fully vented, yielding a joint rotation angle of $55^{\circ}$.

Constant values:
$P a=96 \mathrm{kPa}$
$\mathrm{Pb}=41 \mathrm{kPa}$

Dependent variables:

$\alpha$

\section{Constraints:}

$g_{1}: F_{a} L_{m a}-F_{b} L_{m b}=0$

$g_{2}: 0.03 m \leq L_{1} \leq 0.06 m$

$g_{3}: 0.07 m \leq L_{2} \leq 0.14 m$

$g_{4}: 0.03 m \leq L_{3} \leq 0.06 m$

$g_{5}: 0.07 m \leq L_{a} \leq 0.14 m$

$g_{6}: 0.07 m \leq L_{b} \leq 0.14 m$

$g_{7}: 0 \leq \alpha \leq 90^{\circ}$

Objective function:

Cost $=-\alpha$,

Results : $\alpha_{\max }=-58.4^{\circ}, L_{1}=0.03 \mathrm{~m}, L_{2}=0.1048 \mathrm{~m}$, $L 3=0.06 \mathrm{~m}$.

The joint using these parameters was successful in increasing the workspace of the joint. The new joint had a reachable range of around $\pm 60^{\circ}$, a significant improvement over the previous joint, which maxed out around $\pm 30^{\circ}$. This shows that the analytical modeling and optimization is a useful tool for improving the rPAM driven kinematic linkage skeletons. The introduction of the swivel mounts for the actuators was successful in reducing the bending of the actuators under high angles. The final joint design can be seen in Figure 7.

To show the benefit of the correction force $F_{\text {stretch }}$, we compared the analytical prediction of joint angle with and without $F_{\text {stretch }}$ against the actual results of the fabricated joint. The results, shown in Figure 8, clearly show that the addition of $F_{\text {stretch }}$ makes the analytical model significantly 


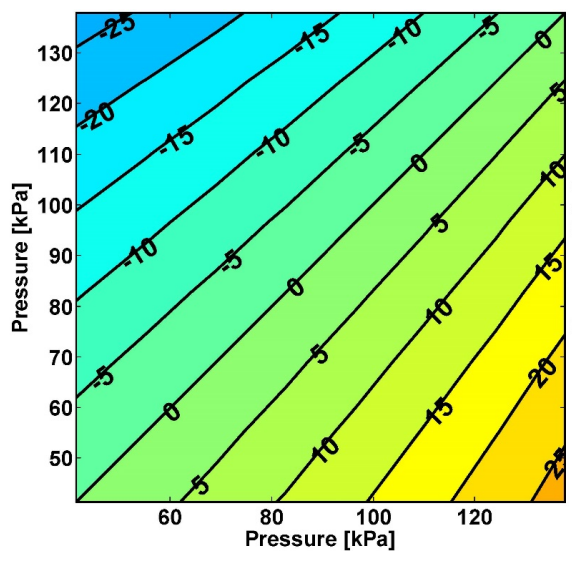

(a) Simulation Without $\boldsymbol{F}_{\text {stretch }}$

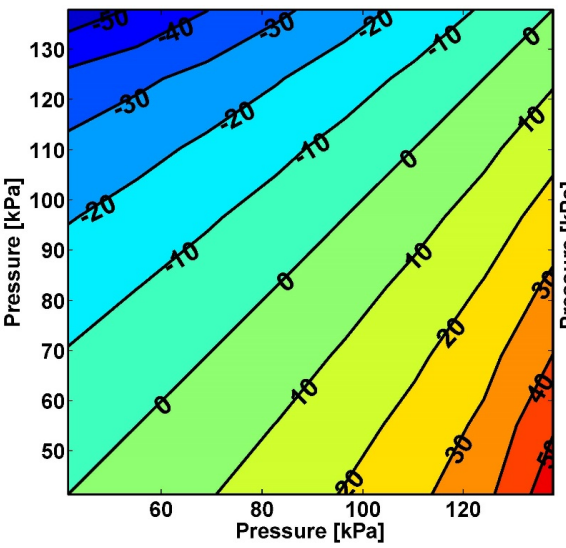

(b) Simulation With $F_{\text {stretch }}$

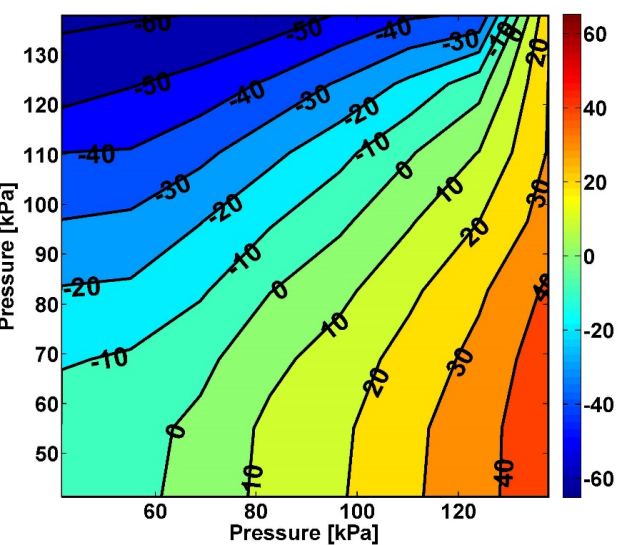

(c) Experiment

Fig. 8. Comparison of model predictions and experimental results for a range of input pressures ranging from 41 to $138 \mathrm{kPa}$ for both rPAMs. Contour plots of the resulting steady-state joint angle in degrees are displayed for new design joint (a) simulation without the initial stretch force, simulation with the initial stretch force and experimental (c) results. Joint angle values are annotated on the curves, and indicated as color coding from blue to red.

more accurate.

\section{CONCLUSION}

In this paper, we refined the analytical model by adding an initial stretch force to better predict the static response of the actuator under large payloads. Additionally, we used this model to perform an optimization on the physical dimensions of the rPAM actuated joint to maximize the joint workspace. We modified joint design to allow it to function at the predicted larger angles, fabricated it, and compared its performance with that of the model. Experimental results showed that the modifications to the model vastly improved its fidelity in predicting joint angles.

However, the method for the initial stretch force calculation is based on empirical data as a means of calibrating the model. Further work needs to be done to find a physical way of representing the constraint force as a function of pressure and load.

In addition, though the swivel mounted actuators allowed the joint to achieve large angles, they had some unforeseen side-effects. Variable friction in the joints increased the probabilistic nature of the final joint angle, as the swivel mounts would not always follow each other accurately. As you can see from Figure 7, the lower right swivel mount has not turned all the way to align itself with the upper right mount. In addition, when both actuators were pressurized equally the swivel mounts combined with the compliant nature of the actuators allowed the joint to the toggle between two separate extreme states where one actuator was fully extended and the other was highly bowed.

We optimized to maximize joint travel alone. Though we succeeded at maximizing this, we did so at the cost of sometime excessive joint compliance. This compliance contributes to the lack of repeatability actuator tests, as the tiny inconsistencies are multiplied to result in a larger amount of variation. This is why there is a larger variation between simulated and experimental with the new joint compared to the original joint. To produce a more useful joint configuration, it would be necessary to optimize a function of both joint angle and stiffness.

For the joint design, our future plans include the development of a 2-DoF shoulder joint driven by three rPAMs. We plan to extend our models to predict the behavior of this type of joint and use them to control it more effectively. We seek to use arrangements of these rigid kinematic systems as part of a robotic arm fully driven by soft linear actuators with controllable stiffness.

\section{REFERENCES}

[1] E. H. Skorina, M. Luo, S. Ozel, F. Chen, W. Tao, and C. D. Onal, "Feedforward augmented sliding mode motion control of antagonistic soft pneumatic actuators," in Robotics and Automation (ICRA), 2015 IEEE International Conference on (under review), IEEE, 2015.

[2] M. Luo, E. H. Skorina, W. Y. Oo, W. Tao, F. Chen, S. Youssefian, N. Rahbar, and C. D. Onal, "Reverse pneumatic artificial muscles: Modeling, analysis, and integration," Soft Matter (under review), 2015.

[3] G. K. Klute, J. M. Czerniecki, and B. Hannaford, "Mckibben artificial muscles: pneumatic actuators with biomechanical intelligence," in Advanced Intelligent Mechatronics, 1999. Proceedings. 1999 IEEE/ASME International Conference on, pp. 221-226, IEEE, 1999.

[4] J. Bishop-Moser, G. Krishnan, and S. Kota, "Force and hydraulic displacement amplification of fiber reinforced soft actuators," in ASME 2013 International Design Engineering Technical Conferences and Computers and Information in Engineering Conference, pp. V06AT07A031-V06AT07A031, American Society of Mechanical Engineers, 2013.

[5] C. D. Onal, X. Chen, G. M. Whitesides, and D. Rus, "Soft mobile robots with on-board chemical pressure generation," in International Symposium on Robotics Research (ISRR), 2011.

[6] C. D. Onal and D. Rus, "A modular approach to soft robots," in Biomedical Robotics and Biomechatronics (BioRob), 2012 4th IEEE $R A S$ \& EMBS International Conference on, pp. 1038-1045, IEEE, 2012.

[7] C. D. Onal and D.Rus, "Autonomous undulatory serpentine locomotion utilizing body dynamics of a fluidic soft robot," Bioinspiration \& biomimetics, vol. 8, no. 2, p. 026003, 2013.

[8] M. Luo, W. Tao, F. Chen, T. K. Khuu, S. Ozel, and C. D. Onal, "Design improvements and dynamic characterization on fluidic elastomer actuators for a soft robotic snake," in Proceedings of the IEEE International Conference on Technologies for Practical Robot Applications, 2014

[9] M. Luo, M. Agheli, and C. D. Onal, "Theoretical modeling and experimental analysis of a pressure-operated soft robotic snake," Soft Robotics, vol. 1, no. 2, pp. 136-146, 2014. 
[10] M. J. Martell and J. A. Schultz, "Multiport modeling of force and displacement in elastic transmissions for underactuated hands," in Intelligent Robots and Systems (IROS 2014), 2014 IEEE/RSJ International Conference on, pp. 1074-1079, IEEE, 2014.

[11] D. W. Robinson, J. E. Pratt, D. J. Paluska, and G. A. Pratt, "Series elastic actuator development for a biomimetic walking robot," in Advanced Intelligent Mechatronics, 1999. Proceedings. 1999 IEEE/ASME International Conference on, pp. 561-568, IEEE, 1999.

[12] J. Pratt, B. Krupp, and C. Morse, "Series elastic actuators for high fidelity force control," Industrial Robot: An International Journal, vol. 29, no. 3, pp. 234-241, 2002.

[13] C. Vergari, P. Pourcelot, L. Holden, B. Ravary-Plumioën, G. Gerard, P. Laugier, D. Mitton, and N. Crevier-Denoix, "True stress and poisson's ratio of tendons during loading," Journal of biomechanics, vol. 44, no. 4, pp. 719-724, 2011.

[14] R. Ogden, "Large deformation isotropic elasticity-on the correlation of theory and experiment for incompressible rubberlike solids," Proceedings of the Royal Society of London. A. Mathematical and Physical Sciences, vol. 326, no. 1567, pp. 565-584, 1972.

[15] Á. Bényi, "87.47 a heron-type formula for the triangle," The Mathematical Gazette, pp. 324-326, 2003. 